\title{
Ann Arbor Lymphoma Staging System
}

National Cancer Institute

\section{Source}

National Cancer Institute. Ann Arbor Lymphoma Staging System. NCI Thesaurus. Code C54179.

The Ann Arbor system classifies lymphoma into four stages based on anatomic lymph nodal group involvement. Disease confined to one nodal group or location defines stage I. Disease limited to one side of the diaphragm, (the muscle separating the chest from the abdomen), defines stage II. Stage III patients have disease on both sides of the diaphragm and stage IV patients once again have disseminated disease. Consideration of involvement of the liver, spleen, and bone marrow are also considered in this system. Finally, the stage is subdivided into categories of A and B depending on the presence of symptoms of itching, weight loss, fever, and night sweats. Those having symptoms receive the designation "B" and have a worse prognosis. 